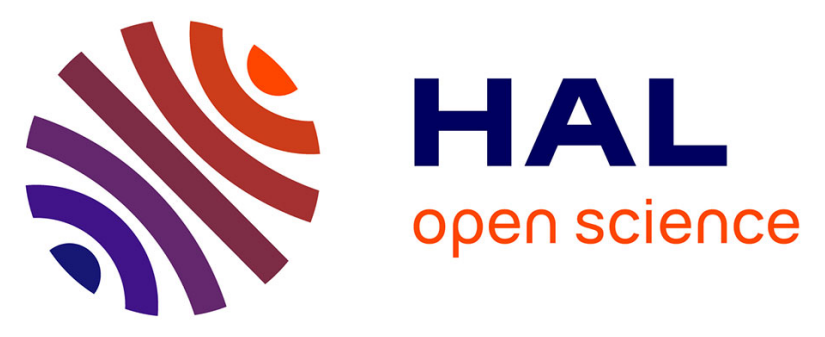

\title{
Rapport final du groupe-projet du Comité pour la Science Ouverte "construire la bibliodiversité"
}

\author{
Jacques Lafait, Jean-François Lutz, Pascal Aventurier, Gégory Colcanap, \\ Jean-Luc de Ochandiano, Thierry Fournier, Julie Giovacchini, Frédéric Hélein, \\ Doriane Ibarra, Denis Jérôme, et al.
}

\section{To cite this version:}

Jacques Lafait, Jean-François Lutz, Pascal Aventurier, Gégory Colcanap, Jean-Luc de Ochandiano, et al.. Rapport final du groupe-projet du Comité pour la Science Ouverte "construire la bibliodiversité".

[Rapport de recherche] Comité pour la science ouverte. 2019, 7 p. hal-03584629

\section{HAL Id: hal-03584629 \\ https://hal.science/hal-03584629}

Submitted on 22 Feb 2022

HAL is a multi-disciplinary open access archive for the deposit and dissemination of scientific research documents, whether they are published or not. The documents may come from teaching and research institutions in France or abroad, or from public or private research centers.
L'archive ouverte pluridisciplinaire HAL, est destinée au dépôt et à la diffusion de documents scientifiques de niveau recherche, publiés ou non, émanant des établissements d'enseignement et de recherche français ou étrangers, des laboratoires publics ou privés.

\section{(c)(1)}

Distributed under a Creative Commons Attribution| 4.0 International License 


\title{
Rapport final du groupe-projet du Comité pour la Science Ouverte
}

\author{
"construire la bibliodiversité"
}

\author{
$0-0-0-0-0-0-0-0-0-0-0$
}

Dans le prolongement de l'Appel de Jussieu publié en octobre 2017, il est apparu nécessaire et urgent de chercher à apporter des solutions à la mise en pratique de la bibliodiversité prônée par l'Appel, tant en France qu'au niveau européen et international. Ces impératifs ont naturellement conduit à la création du groupe-projet "Construire la bibliodiversité" (GPCB) dans le cadre de la fondation du Comité pour la science ouverte (CoSO) via l'AMI du Ministère de la Recherche de mars 2018. Placé sous la responsabilité et le suivi permanent du Collège Publications et du Collège Europe \& International, le GPCB comprenait 47 membres (cercle élargi) dont 21 appartenant au noyau dur travaillant en présentiel. La composition de ce "noyau dur" reflétait bien la diversité des métiers et des disciplines qui, conjuguée à la variété des sensibilités et des points de vue, a contribué à la richesse des échanges et de la production finale. La durée de vie du groupe a été fixée à 9 mois, d'octobre 2018 à juin 2019 avec 9 réunions de travail programmées (10 effectives) ayant réuni en moyenne une quinzaine des membres du noyau dur, avec le co-pilotage de Jacques Lafait et Jean-François Lutz.

Face à l'évolution rapide du paysage de la science ouverte à partir du mois de juillet 2018 (plan français pour la science ouverte présenté par la ministre de la recherche, initiatives de la CURIF, plan S européen, lobbying divers autour de l'élaboration de la nouvelle directive européenne sur le droit d'auteur...), et à la diffusion des solutions assez univoques proposées déjà deux ans auparavant par la Max Planck Gesellschaft avec OA2020, les copilotes ont accéléré la mise en route du groupe-projet et organisé son fonctionnement de manière à le rendre réactif et capable de fournir rapidement des avis et des recommandations au CoSO sur des points brûlants tout en continuant une réflexion de fond sur le développement de la bibliodiversité.

Ces deux aspects du travail - les urgences et le fond - se sont finalement bien complétés et ont maintenu le groupe "sous pression" permanente tout au long de ses neuf mois de mission.

Les objectifs initiaux de définir des principes et des critères de soutien aux initiatives affichant une exemplarité en matière d'accès ouvert et de bibliodiversité, tout comme la nécessité d'établir un cadre pérenne de financement de ces actions ainsi que la participation à toute forme d'échange et d'action au plan international et surtout européen, en vue de promouvoir la bibliodiversité ont en permanence guidé les travaux du GPCB et orienté l'élaboration de ses préconisations. 
La "production" du groupe-projet peut ainsi se regrouper autour de trois thématiques principales : (i) le financement de la science ouverte et les critères d'exemplarité qui s'y rapportent; (ii) les relations internationales et la valorisation des travaux de notre groupe-projet ; (iii) les préconisations à propos du Plan S.

\title{
Critères d'exemplarité des contenus éditoriaux, plateformes et infrastructures pour la science ouverte
}

\begin{abstract}
L'élaboration de ces critères s'est échelonnée d'octobre 2018 jusqu'à leur adoption finale par le Comité de pilotage du CoSO le 17 avril 2019, et a été l'objet de nombreux débats et de plusieurs allers-retours avec le SPSO (Secrétariat permanent de la science ouverte) et avec le Comité de pilotage. Ces critères, au nombre de 42 , doivent prioritairement servir à guider le choix des investissements à réaliser dans le cadre de la politique nationale de science ouverte en général, et du Fonds national pour la science ouverte en particulier. Ils portent sur le degré d'ouverture présent ou en devenir des initiatives et se déclinent notamment en termes de gouvernance, d'éthique et transparence, de viabilité, de réplicabilité/portabilité, de modèle économique, d'interopérabilité. Ils sont classés selon trois niveaux : indispensable, fortement recommandé, souhaité. Ils sont disponibles sur le site Ouvrirlascience du CoSO en versions française et anglaise et seront régulièrement actualisés.
\end{abstract}

\section{Premières orientations pour le fonctionnement du Fonds national pour la Science Ouverte (FNSO) en 2019}

Dans la foulée de l'élaboration des critères d'exemplarité, il a été demandé au GPCB de faire des propositions au CoSO pour le fonctionnement du FNSO annoncé par la ministre de la recherche en juillet 2018. Ces propositions portent sur le périmètre d'action du fonds, les initiatives éligibles (plateformes, livres, revues scientifiques), les critères et la répartition des rôles des acteurs. Elles ont également été validées par le Comité de pilotage du 17 avril 2019.

\section{Propositions pour l'Appel à projet FNSO 2019.}

Ces propositions sont la suite logique du point précédent. Elles précisent dans le détail les conditions et modalités de l'Appel à projet afin qu'il puisse être lancé à l'automne 2019. Elles ont fait l'objet de plusieurs échanges avec le SPSO et la DGRI et ont été soumises au Comité de pilotage du CoSO du 17 juillet 2019. Elles ont été accompagnées d'un projet d'annonce publique de l'Appel à projet.

\section{Projet de financement FNSO à l'international : SCOSS}

Il a été convenu au niveau du CoSO que l'Appel à projet FNSO 2019 serait, pour plus de facilité, strictement national. Par contre, le choix a été fait de consacrer environ $10 \%$ du fonds à des initiatives internationales fiables et reconnues. Le GPCB a rédigé un projet dans ce sens, recommandant SCOSS comme tiers de confiance pour le choix des initiatives à soutenir, accompagné d'une note sur SCOSS destinée au Comité de pilotage du CoSO. 


\section{Relations Appel de Jussieu / OA2020}

Des échanges informels entre les rédacteurs d'OA2020 et de l'Appel de Jussieu avaient eu lieu en vue de dépasser l'antagonisme apparent des deux propositions et mettre en évidence les points de convergence dans l'idée de présenter un front international moins désuni face aux multinationales de l'édition. Notamment à l'occasion de l'invitation de Christoph Bruch par BSN47 (Collège de France, 16/11/2017) et lors des Journées science ouverte Couperin (Paris, 22/01/2018), des rencontres OPERAS (Athènes, 31/05/2018), des Open Access Tage (Graz, 25/09/2018).

OA2020 / Jussieu Call joint statement (02/12/2018). Les journées Berlin 14 (3-4/12/2018) ont fourni l'occasion d'une déclaration officielle commune précisant clairement les spécificités des deux approches (schématiquement : transfert pragmatique des coûts pour lire aux coûts pour publier, en toute transparence, pour OA2020 ; principe de bibliodiversité à appliquer aux modèles éditoriaux et économiques, pas de modèle unique, pour l'Appel de Jussieu) ainsi que la volonté commune, dans la ligne du plan $S$, de soutenir financièrement à parts sensiblement égales les modèles économiques "green", "diamond" et "gold" et de collaborer en 2019 à aligner les "critères open access" à appliquer aux revues, plateformes et infrastructures et à coordonner les investissements à réaliser dans ces domaines.

Cette déclaration, rédigée pour la partie française, en urgence, par les pilotes du GPCB et du Collège publications a été négociée et lue par le représentant du MESRI de la délégation française (Marin Dacos) lors du sommet préparatoire à la conférence. Ce texte est disponible sur le site ouvrirlascience du CoSO et sur le site d'OA2020.

Dans la foulée de la déclaration conjointe Jussieu / OA2020, des contacts ont été pris avec les porteurs du groupe OA2020-DE et une réunion téléphonique s'est tenue le 23 janvier 2019. Les échanges ont permis de mieux comprendre la stratégie de négociation allemande dans le cadre du Projekt DEAL et de disposer d'une présentation des expérimentations de soutien à l'édition ouverte. Le constat d'une différence d'approche assez sensible entre la France et l'Allemagne a été fait à cette occasion, même si les objectifs restent fondamentalement les mêmes.

\section{Communication et valorisation des travaux du GPCB}

Il était entendu dès le départ que les réalisations du groupe, et en particulier les critères d'exemplarité, devaient faire l'objet d'une large diffusion et être présentées lors de conférences internationales et nationales.

Ce fut le cas à trois reprises lors des événements suivants :

- 23e congrès ELPUB à Marseille le 3 juin 2019. Le thème retenu pour ce congrès consacré à l'édition numérique portait précisément sur la bibliodiversité et l'intervention en séance plénière a permis de présenter les travaux du GPCB et tout particulièrement la réflexion menée autour des critères d'exemplarité.

- $\quad 49$ e congrès Liber à Dublin le 28 juin 2019. L'intervention qui s'est déroulée dans le cadre d'une session parallèle consacrée à la Science Ouverte était très proche de celle faite au congrès Elpub mais s'adressait à un public différent, à savoir les responsables de bibliothèques de recherche provenant de différents pays européens.

- 9e journées du réseau Medici à Nantes le 24 septembre 2019. Cette ultime présentation des critères d'exemplarité était destinée aux professionnels de l'édition 
travaillant dans les établissements d'enseignement supérieur et de recherche français.

\section{Préconisations à propos du Plan $\mathrm{S}$}

Dès sa première réunion d'octobre 2018, le GPCB a été sollicité afin de fournir le plus rapidement possible un avis et des préconisations à I'ANR à propos du Plan S. II s'agissait d'éclairer sa prise de position au sein de la cOAlitions chargée d'élaborer et de mettre en application le Plan. L'objectif principal de ce plan étant que les publications scientifiques, qui résultent de recherches qu'elle a financées, soient publiées en accès ouvert dans des revues ou sur des plates-formes à compter du $1^{\text {er }}$ janvier 2020 (date reportée plus tard au $1^{\text {er }}$ janvier 2021) et que les auteurs de ces publications conservent sans aucune restriction leurs droits d'auteur. Les préconisations du GPCB ont porté principalement sur : (1) l'affirmation du principe de bibliodiversité (pas de modèle unique) ; (2) le respect des spécificités des disciplines ; (3) le rôle primordial que doivent jouer les archives ouvertes ; (4) l'exigence de transparence et de mesure des coûts de l'accès ouvert. Ces préconisations élaborées en octobre-novembre 2018 ont été reprises par l'ANR et défendues avec succès auprès de la cOAlitions qui a publié conséquemment le 26 novembre 2018 des orientations sur la mise en œuvre du Plan $S$ et proposé une vaste consultation en ligne appelant à réaction sur ces orientations. Une version remaniée des consignes de mise en oeuvre du Plan $\mathrm{S}$ a été diffusée le 31 mai 2019. Plusieurs des points de vigilance soulignés par le GPCB et de nombreux autres acteurs ont été pris en considération... et la date de mise en application du Plan S a été reculée au $1^{\text {er }}$ janvier 2021.

\section{Points de vigilance pour la mise en œuvre du Plan S}

Le GPCB a donc rédigé une courte note de "points de vigilance" (validée par le SPSO en janvier 2019) en réponse à cette consultation afin de mettre l'accent sur les points éventuellement discutables ou encore imprécis du guide d'implémentation. Les 8 points évoqués reviennent plus précisément sur certaines de nos préconisations (notamment le soutien aux infrastructures, des délais plus larges pour la mise en application du plan et le respect des disciplines...) et recommandent un assouplissement des licences Creative Commons à appliquer aux publications ainsi qu'une aide à apporter aux éditeurs pour la mise en ligne des textes intégraux déposés en archive ouverte dans un format universel de publication .

\section{Travaux à poursuivre et prospective}

Au moment de la clôture programmée de ses travaux, début juillet 2019, et avant la rédaction de ce rapport, le GPCB a tenu à fournir au CoSO les propositions de ses membres (non débattues au sein du groupe) sur des pistes de poursuite de l'action pour la bibliodiversité ainsi que sur les points non réalisés de son programme. 10 thématiques sont ainsi apparues, qui pourraient être reprises par des groupes ou collèges du CoSO ou faire l'objet de la création de groupes-projet à durée déterminée. 
- Degré de conformité des structures existantes aux critères d'exemplarité.

Le passage au crible des critères d'exemplarité d'un certain nombre de plateformes, infrastructures ou structures éditoriales faisait partie du programme que s'était fixé le GPCB et qu'il n'a pas eu le temps de mener à bien. Ce travail mériterait d'être accompli.

- Soutien aux archives ouvertes (formation et infrastructure). Proposition de Julie Giovacchini.

- Mesures d'accompagnement du plan $S$ : soutien pérenne et ciblé à des plateformes de publication sans APC. Proposition de Thierry Bouche.

- Donner de la visibilité aux politiques éditoriales françaises en faveur de l'open access. Proposition d'Armelle Thomas.

- Constitution de fonds numériques structurés standardisés ou normés : pratiques métiers et pratiques disciplinaires, outils, convergences... Proposition de Dominique Roux.

- Suivi de l'évolution d'EDP Sciences par le CoSO et soutien à son évolution vers plus d'ouverture. Proposition de Jacques Lafait.

- Revue de littérature et organisation de la discussion en vue de l'évaluation des accords dits transformants comme leviers pour accélérer le passage à la science ouverte. Proposition de Jean-François Lutz.

- Liens entre publication et données issues de la recherche : comment pouvons-nous accélérer le mouvement associant publication et mise à disposition des données ? Proposition d'Erwin Dreyer.

- La question de la qualité et de la validation des résultats dans les serveurs de preprints et plus généralement dans le cadre du mouvement vers l'accès libre. Proposition d'Erwin Dreyer.

- Le modèle économique de la publication scientifique : comment le faire évoluer vers plus de transparence tout en mobilisant les moyens nécessaires?

Proposition d'Erwin Dreyer.

\section{Conclusion}

Le groupe-projet "Construire la bibliodiversité" étant le premier groupe-projet du CoSO, ses membres ont tenu à respecter le principe de durée de vie limitée de ce type de structure et ont donc clos leurs travaux en juillet 2019 après les neuf mois fixés au départ pour la vie du groupe. Ils ont rodé un mode de fonctionnement collaboratif réactif qui leur a permis de répondre aux demandes urgentes émanant du Secrétariat permanent de la science ouverte. 
II est cependant bien clair que la "construction de la bibliodiversité" dans la ligne de l'Appel de Jussieu est un processus de longue haleine qui devra donc se poursuivre sous d'autres formes et/ou avec des thématiques plus ciblées. Les membres du groupe en ont proposé un certain nombre qui leur paraissaient les plus urgentes ou importantes à traiter. Ils ont aussi affirmé se tenir prêts à participer à la mise en œuvre de nouvelles thématiques en profitant de l'expérience acquise au cours de ces neuf mois.

Les annexes à ce rapport détaillent l'ensemble des textes produits par le groupe-projet au fil de ses neuf mois d'activité (annexe 1) ainsi que la liste des personnes ayant participé aux travaux (annexe 2). 


\section{Annexe 1 : production du groupe-projet "Construire la Bibliodiversité"}

Objectifs fixés au groupe dans le cadre du coso :

- Page du groupe-projet, avec le détail des objectifs, sur le site web du CoSO, février 2018

Textes et documents produits par le Groupe-projet Construire la Bibliodiversité :

- Building Bibliodiversity with Multiple Approaches. Joint Statement of the French Open Science Committee, promotors of the Jussieu Call, and the Open Access 2020 Initiative, 18 décembre 2018

- Préconisations en vue de la mise en oeuvre du Plan S, 26 janvier 2019 (en commun avec le groupe Edition scientifique Ouverte du CoSO), version EN

- Points de vigilance sur le Guide de mise en oeuvre du Plan S, 26 janvier 2019 (en commun avec le groupe Edition scientifique Ouverte du CoSO), version EN

- Critères d'exemplarité en vue de financements par le Fonds national pour la science ouverte via les plateformes, infrastructures et contenus éditoriaux, 17 avril 2019, version EN

- Premières orientations pour le fonctionnement du Fonds National pour la Science Ouverte, document de travail à destination du comité de pilotage du CoSO, juillet 2019

- Travaux à poursuivre et prospective, document de travail à destination du Secrétariat Permanent de la Science Ouverte, juillet 2019

\section{Communications et présentation des travaux :}

- Strengthening bibliodiversity: The current situation in France at national and institutional levels, 23e conférence ELPUB, Marseille, 3 juin 2019

- Building and Funding Bibliodiversity: 1 Year After the Launch of the French Open Science Plan, 48e conférence LIBER, Dublin, 28 juin 2019

- Écosystème de la science ouverte : acteurs et politiques au niveau national et international, 9e journées MEDICI, Nantes, 24 septembre 2019

\section{Annexe 2 : membres du groupe-projet "Construire la Bibliodiversité"}

La liste complète des membres du Groupe-projet se trouve sur le site "ouvrirlascience" à l'adresse : https://www.ouvrirlascience.fr/construire-la-bibliodiversite/

Les membres du "noyau dur" du Groupe-projet :

Copilotes : Jacques Lafait (Sorbonne Université, CNRS) et Jean-François Lutz (Université de Lorraine).

Membres : Pascal Aventurier (IRD), Gégory Colcanap (Université d'Evry Val d'Essonne), Jean-Luc De Ochandiano (Université Jean-Moulin Lyon 3), Erwin Dreyer (INRA), Thierry Fournier (Université de Rennes 1), Julie Giovacchini (CNRS), Frédéric Hélein (Université Paris Diderot), Doriane Ibarra (IFREMER), Denis Jérôme (Académie des Sciences), Benoît Kloeckner (Université Paris-Est Créteil), Isabelle Laboulais (Université de Strasbourg), François-Xavier Mas (Université Paris 13), Jacques Millet (INRIA), Lionel Maurel (INSHSCNRS), Christine Ollendorff (ENSAM), Marie Pellen (OpenEdition), Dominique Roux (Université de Caen Normandie). 